\title{
Fuzzy Functions, Relations, and Fuzzy Transforms (2012)
}

\author{
Salvatore Sessa, ${ }^{1}$ Ferdinando Di Martino, ${ }^{1}$ and Irina G. Perfilieva ${ }^{2}$ \\ ${ }^{1}$ Dipartimento di Costruzioni e Metodi Matematici in Architettura, Università degli Studi di Napoli Federico II, \\ Via Monteoliveto 3, 80134 Napoli, Italy \\ ${ }^{2}$ Centre of Excellence IT4Innovations, Division University of Ostrava, Institute for Research and Applications of Fuzzy Modeling, \\ 30 dubna 22, 70100 Ostrava 1, Czech Republic
}

Correspondence should be addressed to Salvatore Sessa, sessa@unina.it

Received 25 November 2012; Accepted 25 November 2012

Copyright (C) 2012 Salvatore Sessa et al. This is an open access article distributed under the Creative Commons Attribution License, which permits unrestricted use, distribution, and reproduction in any medium, provided the original work is properly cited.

Fuzzy functions, fuzzy relations, and fuzzy transforms are applied to fuzzy systems and like in the first issue published in 2011, here these terms must be intended in general sense. Indeed, the topics concerning fuzzy optimizations with fuzzy functions and relations (including also clustering and PSO algorithms), applications of fuzzy relations/transforms to fusion images, and detection coarse grained fuzzy association rules in the datasets and fuzzy convex processes are dealt with widely in this second issue. The contents of any single paper are resumed in the following sequel:

(i) in the paper of M. Vajgl et al., there is the combination of (at least) two fusion operators; both are based on the F-transform. The first fusion operator is based on a robust partition of the scene domain. The second fusion operator is applied to the residuals of scenes and is based on a finer partition of the same domain. The proposed method can be characterized as a weighted combination of those two and is computationally more effective of the previous algorithms known in the literature;

(ii) in the paper of A.-Q. Li et al., the authors propose to evaluate the production performance of reservoir management units by selecting 12 indicators from the three aspects of production task, production technology, and reservoir development. According to the principle of fuzzy analytic hierarchy process (FAHP), this paper presents a new method to get the weights of these indicators;

(iii) in the paper of A.-B. Hanchevici et al., the authors present a networked control strategy for linear SISO systems affected by variant communication delays.
They adjust the command provided by the PID controller by using fuzzy logic, whose inputs for the fuzzy logic controller (FLC) are represented by the delay and the variation of delay and the output is to adapt the PID controller's command to the new value of the communication. An application is also shown;

(iv) in the first paper of F. D. Martino and S. Sessa, the authors present a new method based on fuzzy transforms for detecting coarse-grained association rules in the datasets. The fuzzy association rules are represented in the form of linguistic expressions and a preprocessing phase determines the optimal fuzzy partition of the domains of the quantitative attributes. The extraction of the fuzzy association rules is executed via the AprioriGen algorithm and a confidence index calculated via the inverse fuzzy transform;

(v) in the paper of M. Matloka, the author presents the notion of $\left(\Phi_{1}, \Phi_{2}\right)$-convex fuzzy processes and some basic properties of such processes, being $\Phi_{1}, \Phi_{2}$ suitable generalized convex-like functions of one or more real variables;

(vi) in the paper of E. Eğrioğlu, the author studies fuzzy time series and proposes a new method based on fuzzy C-means and genetic algorithms for forecasting time invariant series; then he applies this method to several datasets;

(vii) in the second paper of F. D. Martino and S. Sessa, the authors present a method by using the hierarchical cluster based multispecies particle swarm 
optimization to generate a fuzzy system of TagakiSugeno-Kang type encapsulated in a geographical information system considered as environmental decision support for spatial analysis. The spatial area is partitioned in subzones, so the data measured in each subzone are used to extract a fuzzy rule set of the above type.

This second issue continues the editorial lines of the first issue and we hope that it can capture the attention of many readers of the journal whose study can further expand the topics discussed here.

Salvatore Sessa

Ferdinando Di Martino

Irina G. Perfilieva 

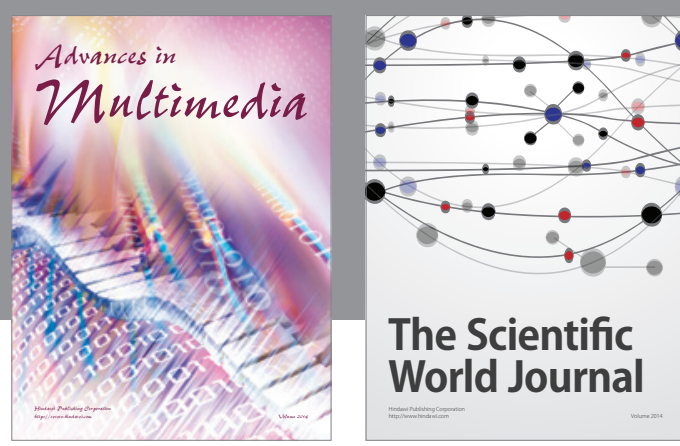

The Scientific World Journal
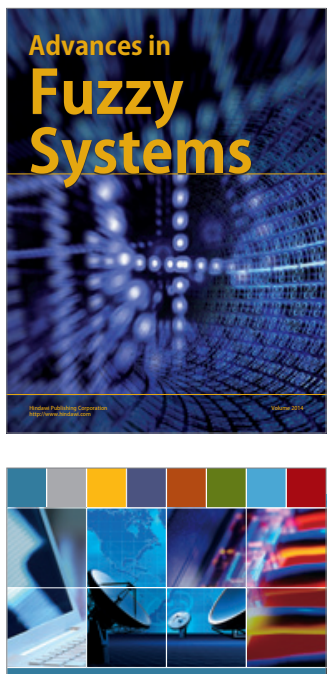

Computer Networks and Communications
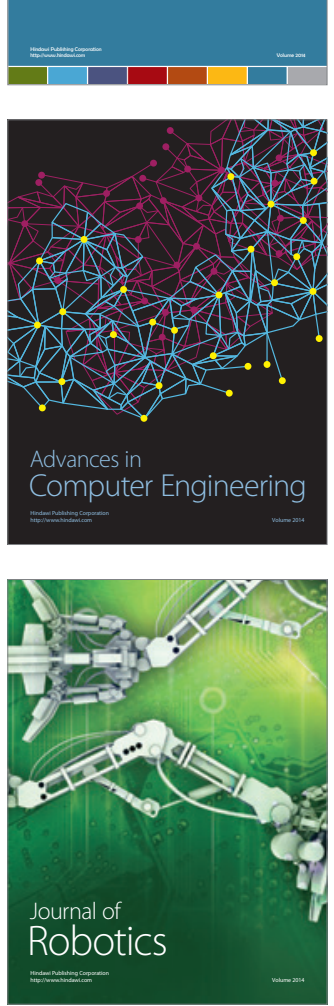
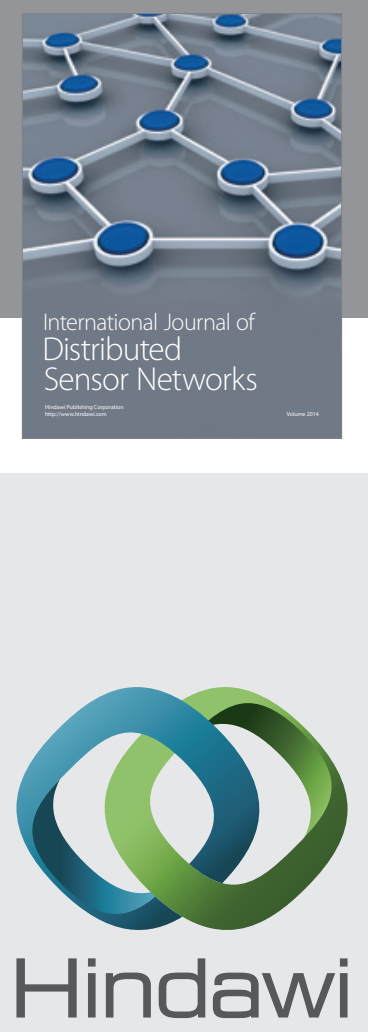

Submit your manuscripts at

http://www.hindawi.com
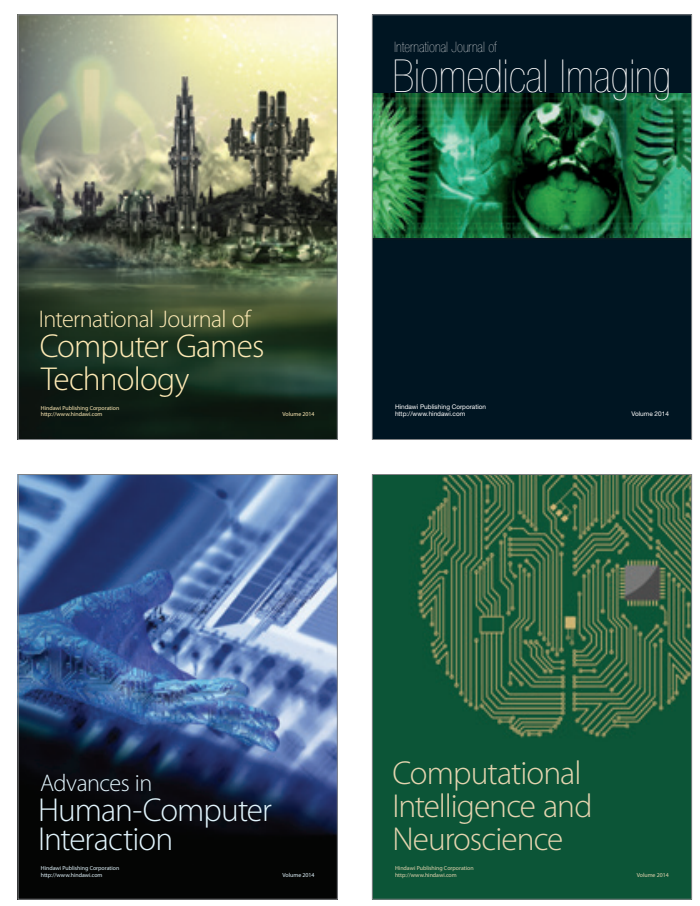
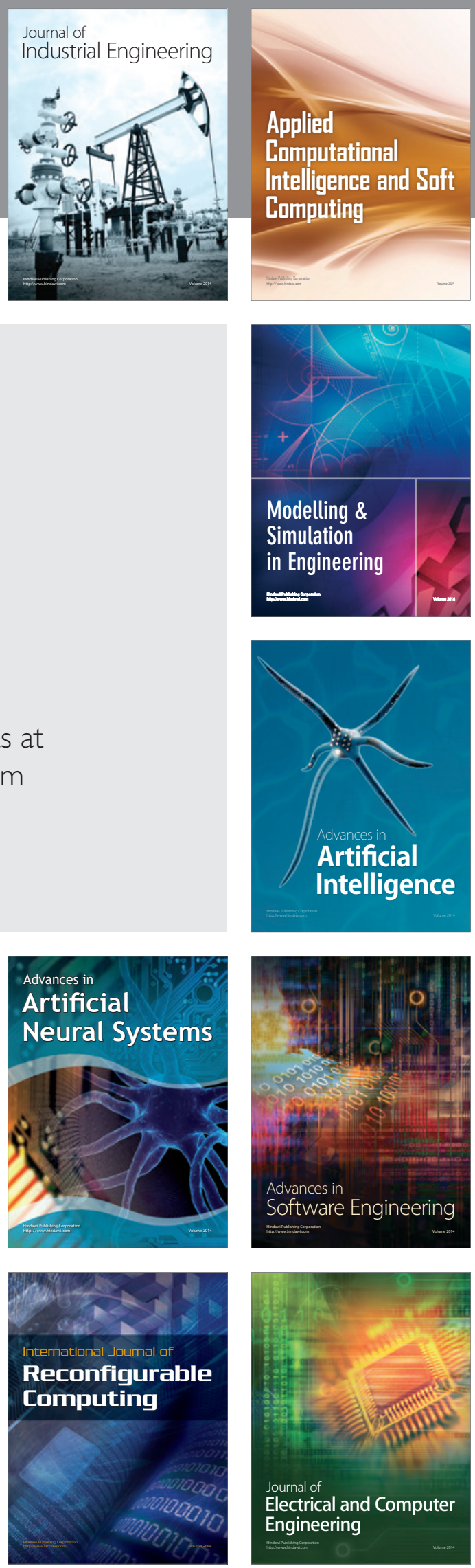